\title{
Inspiratory muscle training in stable COPD patients: enough is enough?
}

\author{
Nicolino Ambrosino \\ Affiliation: Istituti Clinici Scientifici Maugeri, Institute of Montescano (PV), Montescano, Italy.
}

Correspondence: Nicolino Ambrosino, Istituti Clinici Scientifici Maugeri, Institute of Montescano, Via di Montescano, 27040 Montescano (PV), Italy. Email: nico.ambrosinolagmail.com

@ERSpublications

There is enough research to draw conclusions on IMT in COPD patients http://ow.ly/igyD30h9kRE

Cite this article as: Ambrosino N. Inspiratory muscle training in stable COPD patients: enough is enough? Eur Respir J 2018; 51: 1702285 [https://doi.org/10.1183/13993003.02285-2017].

In the sciences, the authority of thousands of opinions is not worth as much as one tiny spark of reason in an individual man

Galileo Galilei

Inspiratory muscle dysfunction is a common finding in patients suffering from chronic obstructive pulmonary disease (COPD), contributing with other factors to dyspnoea and reduced exercise tolerance [1]. Pulmonary rehabilitation programmes (PRPs) including whole-body exercise training improve symptoms, exercise capacity and health-related quality of life (HRQL), and are recommended in all stages of COPD [2, 3]. In 1976, Leith and Bradley [4] were the first to show that the principles of training could be applied also to respiratory muscles: healthy strength trainers significantly increased their maximal static inspiratory and expiratory pressures. Endurance trainers increased their maximal voluntary ventilation but not maximal static pressures.

The joint American College of Chest Physicians/American Association of Cardiovascular and Pulmonary Rehabilitation evidence-based clinical practice guidelines [5] recommended inspiratory muscle training (IMT) in selected COPD patients with dyspnoea and reduced inspiratory muscle strength despite optimal medical therapy. That panel required further randomised controlled trials (RCTs), concluding that "the scientific evidence does not support the routine use of IMT as an essential component of pulmonary rehabilitation" [5]. A later meta-analysis [6] of 32 RCTs with a total sample size of 830 COPD patients found improvements in inspiratory muscle strength and/or endurance, field walking tests, HRQL and dyspnoea in patients performing IMT compared to controls. POLKEY et al. [7] discussed and interpreted the meta-analysis [6] as showing that IMT alone was only "of marginal clinical benefit in COPD". The lack of sham control arms in some studies included in that meta-analysis [6] was also criticised [7]. Again, large studies evaluating patient- centred outcomes rather than, or in addition to, inspiratory muscle function were solicited [7]. The official American Thoracic Society/European Respiratory Society Statement on pulmonary rehabilitation [2] suggested that IMT might be useful when added to a PRP in patients with inspiratory muscle weakness or unable to perform cycling or walking. However, there was another request for further prospective studies [2].

All these dishes that have been ordered are now served in this issue of the European Respiratory Journal. In the single-blind RCT by BEAUmont et al. [8], 149 severe COPD patients were allocated to 4-week PRP

Received: Nov 052017 | Accepted: Nov 082017

Conflict of interest: None declared.

Copyright OERS 2018 
with or without IMT. Dyspnoea was assessed by means of different tools including the recently proposed Multidimensional Dyspnea Profile questionnaire [9]. Despite the expected significant increase in inspiratory muscle strength observed only in the IMT+PRP group, dyspnoea improved significantly in both groups without any significant difference between the two groups. In the RCT by ScHuLTZ et al. [10], 602 COPD patients received a 3-week, inpatient PRP. The intervention group also received IMT whereas the control group received sham IMT. The IMT group showed higher increases in maximal inspiratory pressure and forced inspiratory volume in $1 \mathrm{~s}$, whereas all other assessed outcomes improved significantly in both groups without any significant differences between groups.

It is noteworthy that the total sample size of 751 patients studied in these two RCTs $[8,10]$ taken together accounts for almost the total sample size of all 32 studies eligible for the meta-analysis [6]. In an age of meta-analyses that are not always necessary (in some cases, including only two eligible studies and few patients, perhaps more useful to academic careers than to real progress of knowledge), the work of these authors must be appreciated. In addition, the study by SchulTz et al. [10] used a sham control, without which the placebo effect might influence measures and volitional tests such as self-reported dyspnoea and HRQL, field walking tests, and maximal inspiratory pressure. Patient-centred outcomes were not improved in these two studies. There are several explanations for these results.

1) Were the training protocols used appropriate? In both studies, training method (threshold inspiratory training) and intensity (up to $60 \%$ of maximal inspiratory pressure) were in line with recommendations. However, the IMT protocols involved 3- and 4-week training in the studies by BEAUMONT et al. [8] and Schultz et al. [10] respectively, whereas some studies included in the meta-analysis [6] lasted up to 4 months. Nevertheless, when evaluating a training protocol, the number of sessions might be more relevant than, or equally relevant to, the overall duration [11]. The total number of IMT sessions was 20 and 21 in the studies by BEAumont et al. [8] and Schultz et al. [10] respectively, which is comparable with most studies included in meta-analysis [6].

2) We can argue that potential effects, if any, on patient-centred outcomes of IMT added to a PRP might be hidden by the larger effectiveness of a properly conducted PRP [4].

3) Maybe there is a simpler explanation. Peripheral muscle weakness is observed in stable COPD patients of different Global Initiative for Chronic Obstructive Lung Disease stages [12]: this is the pathophysiological basis of the recognised effective limb muscle exercise training [2]. Contrastingly, the supposed bases of IMT, namely inspiratory muscle weakness and its contribution to a range of clinical outcomes, are questioned, if not denied, in these patients [7].

4) Patient-centred outcomes such as exercise capacity and HRQL, and even survival in COPD, are determined by more factors than inspiratory muscle function. Hyperinflation, comorbidities and nutritional status are some of factors influencing those outcomes [13-15]. None of these conditions can reasonably be modified by IMT.

IMT is a low-cost modality of pulmonary rehabilitation without any known side-effects. Are these advantages enough to prescribe it in COPD patients able to attend a standard PRP? Have these two studies $[8,10]$ said the final word on IMT in these patients? Never say never.

Are there any other applications or should we throw our IMT devices out of the window? The answer can be found in the frame of a suggested personalised PRP [16]. Although the schedule and the maintenance strategy of IMT are still to be evaluated, there are conditions that would potentially benefit from short IMT, such as surgery $[17,18]$ or weaning from mechanical ventilation [19], although, in this case, with some caution, due to a reported, although unclear, increased mortality with IMT [20]. Furthermore, we need more studies of expiratory muscle training, including the abdominal muscles: an increased function of these muscles might potentially be useful for more effective expulsive efforts, such as cough.

In the Internet-based (self) medicine age, the final decision on patient management is the result of several factors: patients' values and preferences, stakeholders' positions, third payers' rules, legal fears, and last (and unfortunately, sometime least) clinical judgment by clinicians. Although squeezed between different positions and interests, clinicians should offer the best personalised care for their individual patients on the basis of the present scientific evidence, appropriately interpreting RCTs, meta-analyses and guidelines $[21,22]$, in addition to their personal experience and necessary empathy.

In conclusion, we must be grateful to the authors of these two studies for making our ideas clearer on a controversial issue such as IMT, or not?

\section{References}

1 O'Donnell DE, Elbehairy AF, Berton DC, et al. Advances in the evaluation of respiratory pathophysiology during exercise in chronic lung diseases. Front Physiol 2017; 8: 82. 
2 Spruit MA, Singh SJ, Garvey C, et al. An official American Thoracic Society/European Respiratory Society statement: key concepts and advances in pulmonary rehabilitation. Am J Respir Crit Care Med 2013; 188: e13-e64.

3 Paneroni M, Simonelli C, Vitacca M, et al. Aerobic exercise training in very severe chronic obstructive pulmonary disease: a systematic review and meta-analysis. Am J Phys Med Rehabil 2017; 96: 541-548.

4 Leith DE, Bradley M. Ventilatory muscle strength and endurance training. J Appl Physiol 1976; 41: 508-516.

5 Ries AL, Bauldoff GS, Carlin BW, et al. Pulmonary rehabilitation: joint ACCP/AACVPR evidence-based clinical practice guidelines. Chest 2007; 131: 4-42.

6 Gosselink R, De Vos J, van den Heuvel SP, et al. Impact of inspiratory muscle training in patients with COPD: what is the evidence? Eur Respir J 2011; 37: 416-425.

7 Polkey MI, Moxham J, Green M. The case against inspiratory muscle training in COPD. Eur Respir J 2011; 37: 236-237.

8 Beaumont M, Mialon P, Le Ber C, et al. Effects of inspiratory muscle training on dyspnoea in severe COPD patients during pulmonary rehabilitation: controlled randomised trial. Eur Respir J 2018; 51: 1701107.

9 Morélot-Panzini C, Gilet H, Aguilaniu B, et al. Real-life assessment of the multidimensional nature of dyspnoea in COPD outpatients. Eur Respir J 2016; 47: 1668-1679.

10 Schultz K, Jelusic D, Wittmann M, et al. Inspiratory muscle training does not improve clinical outcomes in 3-week COPD rehabilitation: results from a randomised controlled trial. Eur Respir J 2018; 51: 1702000.

11 Clini E, Foglio K, Bianchi L, et al. In-hospital short-term training program for patients with chronic airway obstruction. Chest 2001; 120: 1500-1505.

12 Seymour JM, Spruit MA, Hopkinson NS, et al. The prevalence of quadriceps weakness in COPD and the relationship with disease severity. Eur Respir J 2010; 36: 81-88.

13 Casanova C, Aguirre-Jaime A, de Torres JP, et al. Longitudinal assessment in COPD patients: multidimensional variability and outcomes. Eur Respir J 2014; 43: 745-753.

14 Divo MJ, Casanova C, Marin JM, et al. COPD comorbidities network. Eur Respir J 2015; 46: 640-650.

15 Prospective Studies Collaboration. Body-mass index and cause-specific mortality in 900000 adults: collaborative analyses of 57 prospective studies. Lancet 2009; 373: 1083-1096.

16 Ambrosino N, Clini EM. Response to pulmonary rehabilitation: toward personalised programmes? Eur Respir J 2015; 46: 1538-1540.

17 Brunelli A, Charloux A, Bolliger CT, et al. ERS/ESTS clinical guidelines on fitness for radical therapy in lung cancer patients (surgery and chemo-radiotherapy). Eur Respir J 2009; 34: 17-41.

18 Ambrosino N, Gabbrielli L. Physiotherapy in the perioperative period. Best Pract Res Clin Anaesthesiol 2010; 24: 283-289.

19 Elkins M, Dentice R. Inspiratory muscle training facilitates weaning from mechanical ventilation among patients in the intensive care unit: a systematic review. J Physiother 2015; 61: 125-134.

20 Bissett BM, Leditschke IA, Neeman T, et al. Inspiratory muscle training to enhance recovery from mechanical ventilation: a randomised trial. Thorax 2016; 71: 812-819.

21 Karanicolas PJ, Kunz R, Guyatt GH. Point: evidence-based medicine has a sound scientific base. Chest 2008; 133 : 1067-1071.

22 Tobin MJ. Counterpoint: evidence-based medicine lacks a sound scientific base. Chest 2008; 133: 1071-1074. 